\title{
Inducing and optimizing magnetism in graphene nanomeshes
}

\author{
Hong-Xin Yang and Mairbek Chshiev* \\ SPINTEC, CEA/CNRS/UJF-Grenoble 1/Grenoble-INP, INAC, FR-38054 Grenoble, France
}

Danil W. Boukhvalov

School of Computational Sciences, Korea Institute for Advanced Study (KIAS), Hoegiro 87, Dongdaemun-Gu, Seoul 130-722, Korean Republic

Xavier Waintal

SPSMS-INAC-CEA, 17 rue des Martyrs, FR-38054 Grenoble, France

Stephan Roche

CIN2 (ICN-CSIC) and Universitat Autonoma de Barcelona, Catalan Institute of Nanotechnology, Campus de la UAB, ES-08193 Bellaterra (Barcelona), Spain and ICREA, Institució Catalana de Recerca i Estudis Avancats, ES-08010 Barcelona, Spain

(Received 14 November 2011; published 2 December 2011)

\begin{abstract}
Using first-principles calculations, we explore the electronic and magnetic properties of graphene nanomesh (GNM), a regular network of large vacancies, produced either by lithography or nanoimprint. When removing an equal number of $A$ and $B$ sites of the graphene bipartite lattice, the nanomesh made mostly of zigzag (armchair) -type edges exhibit antiferromagnetic (spin unpolarized) states. In contrast, in situations of sublattice symmetry breaking, stable ferri(o)magnetic states are obtained. For hydrogen-passivated nanomesh, the formation energy is dramatically decreased, and ground state is found to strongly depend on the vacancies shape and size. For triangular-shaped holes, the obtained net magnetic moments increase with the number difference of removed $A$ and $B$ sites in agreement with Lieb's theorem for even $A+B$. For odd $A+B$ triangular meshes and all cases of nontriangular nanomeshes, including the one with even $A+B$, Lieb's theorem does not hold anymore, which can be partially attributed to the introduction of armchair edges. In addition, large triangular-shaped GNMs could be as robust as nontriangular GNMs, providing a possible solution to overcome one of the crucial challenges for the $s p$ magnetism. Finally, significant exchange-splitting values as large as $\sim 0.5 \mathrm{eV}$ can be obtained for highly asymmetric structures evidencing the potential of GNM for room-temperature carbon-based spintronics. These results demonstrate that a turn from zero-dimensional graphene nanoflakes throughout one-dimensional graphene nanoribbons with zigzag edges to GNM breaks localization of unpaired electrons and provides deviation from the rules based on Lieb's theorem. Such delocalization of the electrons leads the switch of the ground state of a system from an antiferromagnetic narrow gap insulator discussed for graphene nanoribons to a ferromagnetic or nonmagnetic metal.
\end{abstract}

DOI: 10.1103/PhysRevB.84.214404

PACS number(s): 81.05.ue, 75.70.-i, 75.75.-c

\section{INTRODUCTION}

Two-dimensional graphene has emerged as a natural candidate for developing "beyond CMOS" nanoelectronics. ${ }^{1-6}$ In addition to the reported huge charge mobilities, the weak intrinsic spin-orbit coupling in carbon-based $s p^{2}$ structures ${ }^{7,8}$ could potentially allow for very large (micrometer-long) spin diffusion lengths. These features, together with the other "semiconductorlike" properties of graphene, make graphenebased spintronic devices highly promising 9,10 and have triggered a quest for controlling spin injection in graphene. ${ }^{11-14}$ Many routes have been attempted to induce magnetism by proximity effect or inject spins from magnetic electrodes. ${ }^{15}$ Another, more intrinsic, possibility is shaping the geometry of graphene by designing graphene nanoribbons (GNR) with zigzag edges. ${ }^{16,17}$ This has been found to induce localized edge magnetic states which can serve as a conceptually new building block for spintronics. ${ }^{18-21}$

The existence of intrinsic magnetism driven by atomicscale defects (such as vacancies, chemisorbed species, grain boundaries, etc.) has also been suggested theoretically, ${ }^{20,22-30}$ but remains fiercely debated on the experimental side. ${ }^{31}$ It is indeed particularly difficult to achieve a precise experimental characterization of those defects, whereas the control of their density, positioning, or chemical reactivity seems an insurmountable challenge, jeopardizing a further use of magnetic properties in real devices. Additionally, the absence of a true energy gap in two-dimensional graphene limits the elaboration of active graphene-based devices and circuits with standard semiconductor technologies.

Another route to make graphene magnetic is either chemisorption of an odd number of adatoms or functional groups, ${ }^{20,32}$ or using magnetism on zigzag edges. ${ }^{18,33-35}$ In the first case the stability of magnetic configurations at room temperature can be easily destroyed by the migration of adatoms with turning the system into nonmagnetic configuration. ${ }^{32}$ In contrast to the adatom-based magnetism edge ${ }^{34}$ and vacancy, ${ }^{36}$ magnetism in graphite is stable at room temperature. But herewith localization of the magnetic moments on the edges provides formation of the antiferromagnetic (AFM) exchange interactions between two edges. ${ }^{18}$

The case of graphene nanoribbons obeys Lieb's theorem because the localized electrons on one edge belong to sublattice $A$ and localized electrons from the other edge belong to sublattice $B$. Magnetism on the edges of graphene nanoflakes is also 
described by this theorem. ${ }^{37}$ Electron localization plays an important role in the different many-body effects on graphene edges $^{38}$ and bulk graphene. ${ }^{39}$ Understanding the nature of the electron localization and delocalization in graphene and related systems is necessary not only for control and manipulation of magnetism in studied compounds but also for the development of knowledge about systems with strongly correlated electrons.

Graphene nanomesh (GNM) is the intermediate compound between graphene nanoribbons with localized electrons on zigzag edges and perfect bulk graphene with delocalized electrons. The fabrication of GNM, using block copolymer lithography and offering versatility in varying periodicities and neck widths down to $5 \mathrm{~nm},{ }^{40}$ could circumvent the hurdles. Indeed, such technique allows a scalable engineering of superlattices of large graphene vacancies whose density, shape, and distribution can be controlled down to the nanoscale. Additionally, GNM-based field-effect transistors were shown to withstand current densities two orders of magnitudes larger than individual graphene nanoribbon devices, with comparable on/off ratio and easily tunable by varying the neck width. Moreover, in accordance to the well-established Lieb's theorem, ${ }^{41}$ the possibility to control inner edge structures ${ }^{42}$ of nanomesh vacancies could enable a true control of intrinsic magnetic properties.

In this paper, we present first-principles calculations of electronic and magnetic properties of graphene nanomesh. We found that by varying the shape, different types of intrinsic ferrimagnetic ${ }^{43}$ states can be obtained with clear identification of optimal conditions. Systematic studies of nonpassivated and hydrogen-passivated GNM are achieved by varying the difference $\left(\Delta_{A B}=|B-A|\right)$ between missing $A$ and $B$ sites of the underlying bipartite lattice and analyzing different hole geometries. For nonpassivated GNM with $\Delta_{A B}=0$, stable nonmagnetic states are found for armchair edge termination, while zigzag edge terminations result in antiferromagnetic ground states. These localized magnetic moments, however, vanish when all edge $C$ atoms are hydrogen passivated. In sharp contrast, when $\Delta_{A B} \neq 0$, stable ferri(o)magnetic states are induced with net moments up to $4 \mu_{B}$ (per 6 $\times 6$ unit cell) originating from dangling bonds of edge atoms.

Furthermore, for hydrogen-passivated GNM, the formation energy is dramatically decreased, and ground state is found to strongly depend on the vacancies shape and size. Our calculations reveal the existence of three magnetic regimes which depend on $\Delta_{A B}$ : (i) highly magnetic GNMs obeying Lieb's theorem corresponding to triangularshaped holes with even $A+B$; (ii) GNMs with quenched magnetic state due to complete chemical bond reconstruction with $\Delta_{A B}=1$ and trivially nonmagnetic state with $\Delta_{A B}=0$; and (iii) GNMs following intermediate regime between magnetic and quenched magnetic states, i.e. triangular GNMs with odd $A+B$ and more complicated structures including both even (e.g., sector-shaped GNM) and odd (e.g., pentagon-shaped GNM) $A+B$. We show that large triangular GNMs could be as robust as nontriangular GNMs, providing a possible solution to overcome one of the crucial challenges for the $s p$ magnetism. Moreover, significant exchange-splitting values as large as $\sim 0.5 \mathrm{eV}$ can be obtained

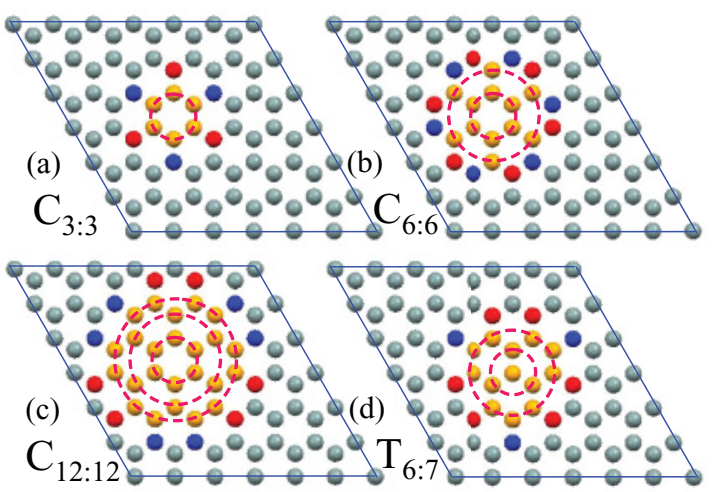

FIG. 1. (Color online) [(a)-(c)] Schematics of the calculated crystalline structures for balanced nonpassivated circular-shaped $\mathrm{C}_{3: 3}$, $\mathrm{C}_{6: 6}$, and $\mathrm{C}_{12: 12} \mathrm{GNM}$ structures, respectively; (d) the same for unbalanced nonpassivated triangular-shaped $\mathrm{T}_{6: 7}$ GNM structure. Edge carbon atoms are in blue and red to represent $A$ and $B$ sites, respectively. For convenience, positions of removed atoms are indicated in orange.

for highly asymmetric structures evidencing the potential of graphene nanomesh for room-temperature carbon-based spintronics.

\section{METHODS}

First-principles calculations were performed using the Vienna $a b$ initio simulation package (VASP) ${ }^{44}$ based on density functional theory (DFT) with generalized gradient approximation (GGA) for exchange-correlation potential. We have used the projected augmented wave (PAW) method ${ }^{45}$ with the Perdew-Becke-Ernzerhof (PBE) parametrization ${ }^{46}$ potentials to describe the core electrons of carbon. Periodic $6 \times 6$ unit cells were used to simulate nonpassivated GNM structures as shown in Fig. 1, whereas periodic $8 \times 8$ unit cells were used to simulate H-passivated GNM structures. The kinetic cutoff energies for the plane-wave basis set used to expand the Kohn-Sham orbitals were $520 \mathrm{eV}$ for the self-consistent energy calculations. The Methfessel-Paxton method $^{47}$ is used with a broadening width of $0.2 \mathrm{eV}$ for the partial occupancy smearing calculations. A $9 \times 9 \times 1 k$-point mesh was sufficient to ensure good convergence in the total energy differences. The structural relaxations were performed ensuring that the Hellmann-Feynman forces acting on ions were less than $10^{-3} \mathrm{eV} / \AA$.

\section{MODEL OF GRAPHENE NANOMESH}

A GNM can be formed by either removing atoms centered of a six ring structure [Figs. 1(a)-1(c)] or centered of one carbon atom [Fig. 1(d)], which leads to the formation of GNM with eihter a balanced or an unbalanced number of removed $A$ and $B$ sites. For the sake of clarity, we label those structures according to their shapes and put the number of removed $A$ and $B$ - site atoms as a subscript. For instance, the structure of Fig. 1(a) named $\mathrm{C}_{3: 3}$, corresponds to a circle hole shape GNM with 3:3 denoting three $A$ and three $B$ atoms removed 
TABLE I. The number of removed atoms on $A$ and $B$ sites and their difference $\Delta_{A B}$. Magnetic moment $M\left(\mu_{B}\right)$, total energies $E$ (in eV) for ferri(o)magnetic (Ferri(o)), antiferromagnetic (AF), and paramagnetic (PM) states, and defect formation energies $E_{f}$ (in eV) for different GNM structures. The total energies for ground states of different structures are specified in bold. The nonpassivated $\mathrm{C}_{3: 3}, \mathrm{C}_{6: 6}, \mathrm{C}_{12: 12}$, and $\mathrm{T}_{6: 7}$ and passivated $\mathrm{C}_{12: 12}^{\mathrm{H}}$ GNMs are calculated with a $6 \times 6$ unit cell; others are calculated with an $8 \times 8$ unit cell.

\begin{tabular}{|c|c|c|c|c|c|c|}
\hline \multirow[b]{2}{*}{ Structure } & \multirow[b]{2}{*}{$\Delta_{A B}$} & \multirow[b]{2}{*}{$M\left(\mu_{B}\right)$} & \multirow[b]{2}{*}{ PM } & \multicolumn{2}{|l|}{$E_{\text {total }}(\mathrm{eV})$} & \multirow[b]{2}{*}{$E_{f}(\mathrm{eV} / \mathrm{C})$} \\
\hline & & & & $\mathrm{AF}$ & Ferri(o) & \\
\hline $\mathrm{C}_{3: 3}$ & 0 & 0 & -590.414 & -591.742 & $(-591.185)$ & 2.81 \\
\hline $\mathrm{C}_{6: 6}$ & 0 & 0 & -528.112 & Unstable & Unstable & 2.10 \\
\hline $\mathrm{C}_{12: 12}$ & 0 & 0 & -406.137 & -408.953 & $(-407.659)$ & 1.402 \\
\hline $\mathrm{T}_{6: 7}$ & 1 & 3.99 & -516.732 & Unstable & $\begin{array}{c}-\mathbf{5 1 8 . 2 5 5} \\
(-517.630)\end{array}$ & 1.98 \\
\hline $\mathrm{T}_{6: 7}^{\mathrm{H}}$ & 1 & $10^{-4}$ & -1089.046 & Unstable & -1089.046 & 0.142 \\
\hline $\mathrm{T}_{10: 12}^{\mathrm{H}}$ & 2 & 1.80 & -1015.387 & Unstable & -1015.403 & 0.120 \\
\hline $\mathrm{T}_{15: 18}^{\mathrm{H}}$ & 3 & 2.16 & -923.346 & Unstable & -923.365 & 0.103 \\
\hline $\mathrm{T}_{21: 25}^{\mathrm{H}}$ & 4 & 3.62 & -813.104 & Unstable & -813.223 & 0.084 \\
\hline $\mathrm{S}_{19: 21}^{\mathrm{H}}$ & 2 & 1.04 & -862.346 & Unstable & -862.348 & 0.082 \\
\hline $\mathrm{P}_{18: 21}^{\mathrm{H}}$ & 3 & 2.15 & -872.125 & Unstable & -872.147 & 0.156 \\
\hline $\mathrm{C}_{3: 3}^{\mathrm{H}}$ & 0 & 0 & -627.565 & Unstable & Unstable & 0.22 \\
\hline $\mathrm{C}_{12: 12}^{\mathrm{H}}$ & 0 & 0 & -481.358 & Unstable & Unstable & 0.078 \\
\hline $\mathrm{R}_{24: 24}^{\mathrm{H}}$ & 0 & 0 & -795.267 & Unstable & Unstable & 0.070 \\
\hline $\mathrm{P}_{18: 19}^{\prime \mathrm{H}}$ & 1 & $10^{-4}$ & -887.147 & Unstable & -887.147 & 0.075 \\
\hline
\end{tabular}

from perfect graphene. The superscript $\mathrm{H}$ is used for hydrogenpassivated GNM.

\section{RESULTS AND DISCUSSIONS}

For the $\mathrm{C}_{3: 3}$ structure [Fig. 1(a)], we find that the configuration with opposite spin orientation between adjacent edge $\mathrm{C}$ atoms is energetically favored in comparison with the configuration with parallel spins between edge atoms of two sublattices represented by blue and red in Fig. 1. The total energy calculations reveal quite large magnetic interaction energies. For instance, the energy difference between a ferromagnetic (FM) spin-polarized and a paramagnetic (PM) state is found to be $0.129 \mathrm{eV}$ per edge atom. The spin configuration is further stabilized by $0.093 \mathrm{eV}$ per edge atom as a result of the antiferromagnetic (AF) coupling between neighboring atoms with a magnetic moment of $0.48 \mu_{B}$ per edge atom for each spin on each sublattice with opposite orientation. The magnetic moment is slightly larger than that of graphene nanoribbons, which is $\sim 0.43 \mu_{B} .{ }^{18}$

We now discuss the case of a three-ring defect $\mathrm{C}_{12: 12} \mathrm{GNM}$ [Fig. 1(c)]. The total energy calculations show that the ground state is AF with a magnetic moment of $0.45 \mu_{B}$ per edge atom for each spin on each sublattice with opposite orientation. The FM state is lower by $0.127 \mathrm{eV}$ per edge atom compared to the PM state, and the spin configuration is further stabilized by $0.107 \mathrm{eV}$ per edge atom as a result of the AF coupling between neighboring atoms on different sublattices with opposite spin orientations (see Table I).

The one-ring and three-ring defect $\mathrm{C}_{3: 3}$ and $\mathrm{C}_{12: 12}$ structures considered above present no net permanent magnetic moment, since the spin-polarized edge atoms appear in pairs with opposite orientations, resulting in an AF ground state with balanced spin-up and spin-down sublattices. However, in view of spintronic applications, it would be much more interesting to find the GNM structures with nonzero net magnetic moment. This can actually be done by building an unbalanced sublattice [Fig. 1(d)]. We found that the ground state of this unbalanceddefect triangular structure $\mathrm{T}_{6: 7}$ turns out to be ferrimagnetic

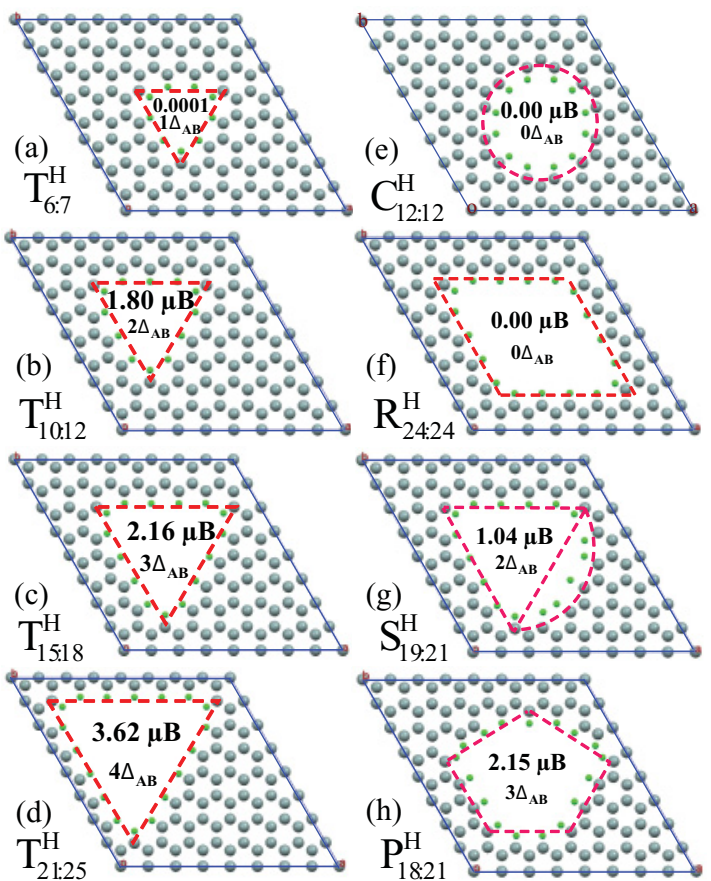

FIG. 2. (Color online) H-passivated GNMs with triangular shapes: (a) $\mathrm{T}_{6: 7}^{\mathrm{H}}$, (b) $\mathrm{T}_{10: 12}^{\mathrm{H}}$, (c) $\mathrm{T}_{15: 18}^{\mathrm{H}}$, (d) $\mathrm{T}_{21: 25}^{\mathrm{H}}$; with a circular shape: (e) $\mathrm{C}_{12: 12}^{\mathrm{H}}$; with a rhombic shape: (f) $\mathrm{R}_{24: 24}^{\mathrm{H}}$; with a sector shape: (g) $\mathrm{S}_{19: 21}^{\mathrm{H}}$; and with a pentagon shape: (h) $\mathrm{P}_{18: 21}^{\mathrm{H}}$. The corresponding net magnetic moments for each structure are also indicated. 


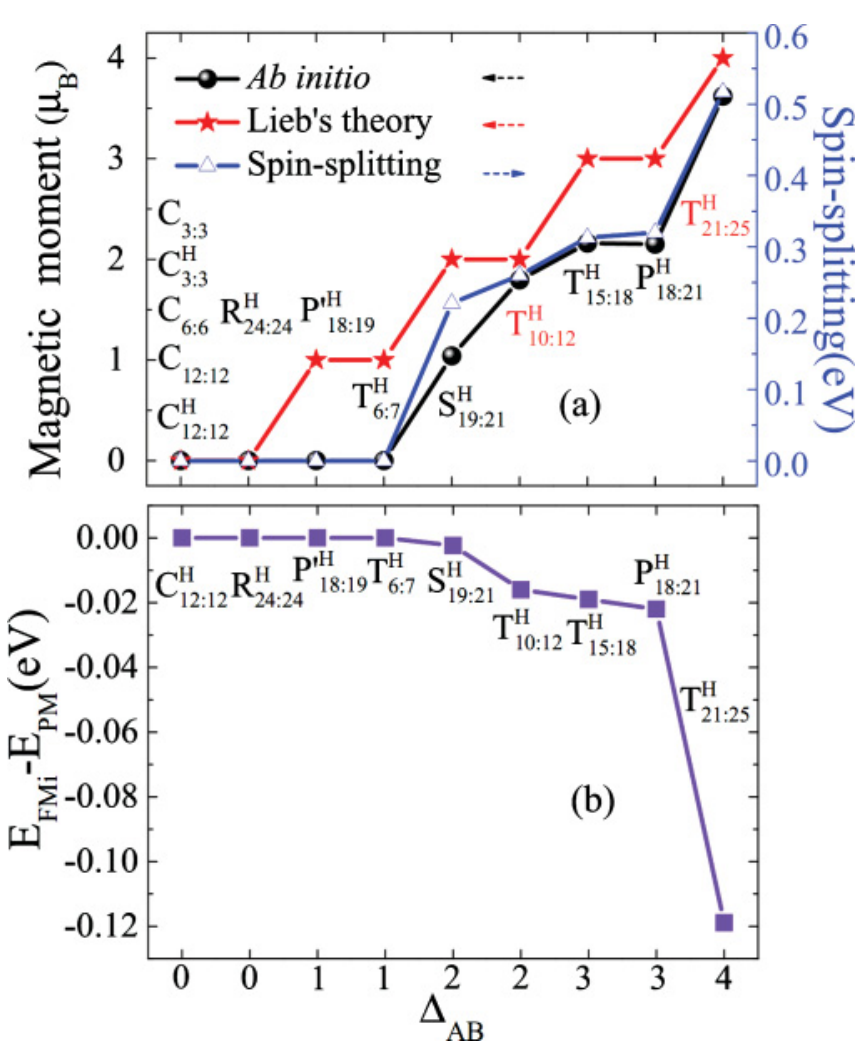

FIG. 3. (Color online) (a) Total magnetic moment ( $\mu_{B} /$ cell) (left) and spin-splitting (right) as a function of $\Delta_{A B}$ for various GNM geometries, where $\mathrm{P}_{18: 19}^{\prime \mathrm{H}}$ is transformed from a pentagon structure $\left(\mathrm{P}_{18: 21}^{\mathrm{H}}\right)$ by adding two $A$ atoms to the two opened hexagons. The result of the Lieb's theorem prediction is also given for comparison. The even number of $A+B$ structures $\mathrm{T}_{10: 12}^{\mathrm{H}}$ and $\mathrm{T}_{21: 25}^{\mathrm{H}}$ are shown in red to indicate the good agreement with Lieb's theorem prediction. (b) Energy difference between ferrimagnetic and paramagnetic states.

(FMi) with a total net moment of $3.987 \mu_{B}$ per unit cell. This moment originates from each edge atom's dangling bond ( $\sigma$ bond) with a spin moment of $1 \mu_{B}$ providing the magnetic moment of 6 (red) -3 (blue) $=3 \mu_{B}$ in addition to a contribution from the $\pi$ bond equal to $1 \mu_{B}$ according to the total number difference between atoms on the $A$ and $B$ sublattices $\Delta_{A B}$.

Dangling bonds at the edge $\mathrm{C}$ atoms of nonpassivated GNM are strongly chemically reactive, ${ }^{48}$ which leads to a hole formation energy of pure GNM higher than $1 \mathrm{eV} / \mathrm{C}$ (see Table I). Thus edge $\mathrm{C}$ atoms are likely to be passivated by light elements. We used hydrogen for passivation of edge $\mathrm{C}$ atoms and considered basic geometrical GNM shapes (Fig. 2) for which the GNM hole formation energy is dramatically decreased due to passivation of dangling bonds (see Table I). For triangular holes, one can see that the formation energy decays as a function of hole dimension. At the same time, the magnetic moment increases and is roughly proportional to the GNM hole size. When the triangular hole size is increased, one observes that the net moment gets bigger [Figs. 2(b)-2(d)] and reaches $3.62 \mu_{B}$ for the biggest hole shown in Fig. 2(d). It is interesting to note that Lieb's theorem was originally formulated for an even $A+B$ number of atoms, and indeed the obtained values for $\mathrm{T}_{10: 12}^{\mathrm{H}}$ and $\mathrm{T}_{21: 25}^{\mathrm{H}}$ follow Lieb's theorem predictions. However, there is an exception for the nontriangular case of $\left(\mathrm{S}_{19: 21}^{\mathrm{H}}\right)$ with a total sum of $A$ and $B$ being even, which is not well accounted by Lieb's theorem. In addition, one can see from Table I that the formation energy values of triangular GNMs decrease as a function of hole size and are comparable to those of the nonmagnetic configurations.

In Fig. 3(a) we summarize the aforementioned results including the calculated net magnetic moments for circular $\left(\mathrm{C}_{12: 12}^{\mathrm{H}}\right)$, rhombic $\left(\mathrm{R}_{24: 24}^{\mathrm{H}}\right)$, sector $\left(\mathrm{S}_{19: 21}^{\mathrm{H}}\right)$, and pentagon $\left(\mathrm{P}_{18: 21}^{\mathrm{H}}\right)$ GNM shapes represented in Figs. 2(e)-2(h), respectively. In addition, the curves contain the net magnetic moment values for alternative pentagon-shape GNM, $\mathrm{P}_{18: 19}^{\mathrm{H}}$, obtained from $\mathrm{P}_{18: 21}^{\mathrm{H}}$ by adding two $A$ sites to complete two hexagons in the

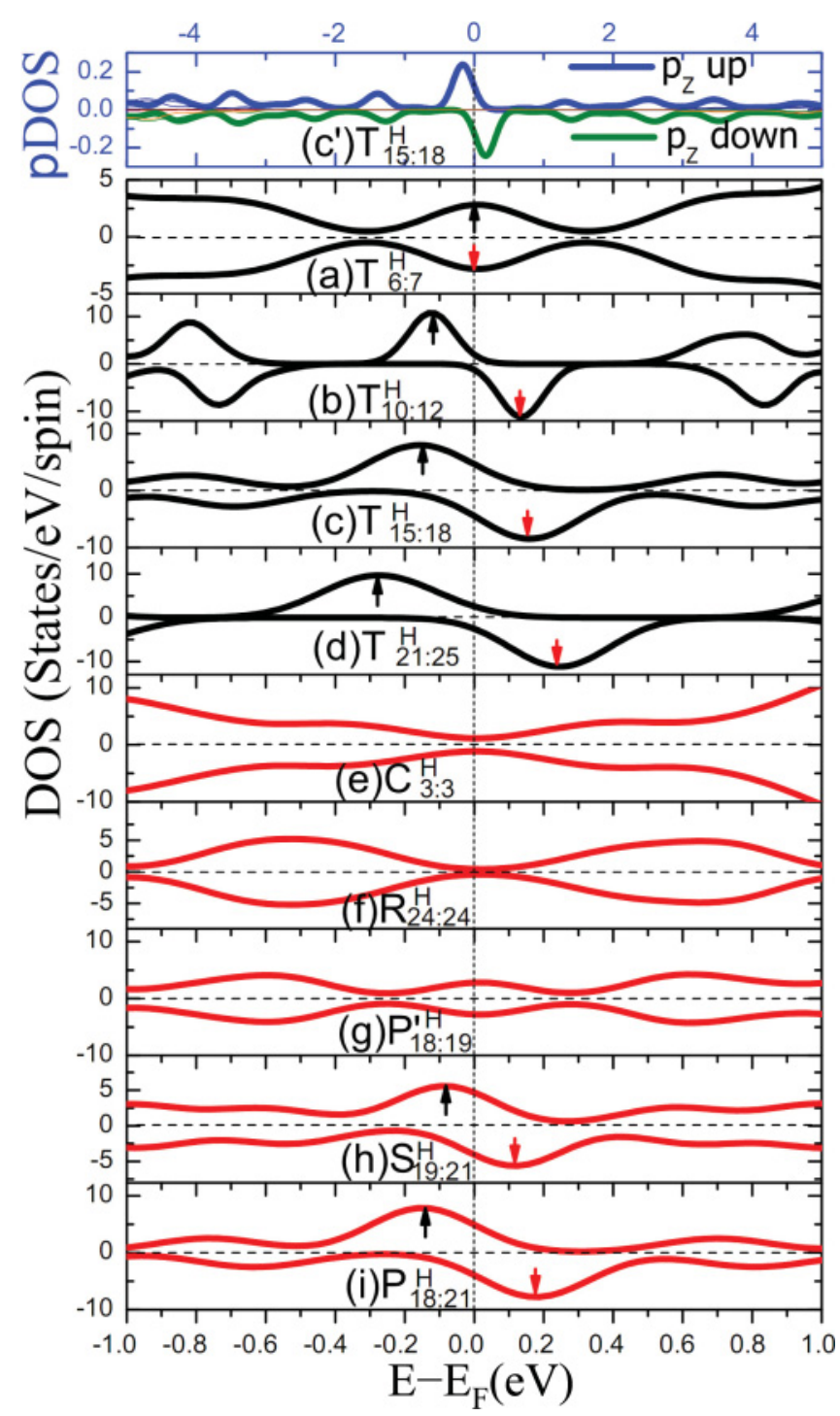

FIG. 4. (Color online) Density of states for triangular GNMs of (a) $\mathrm{T}_{6: 7}^{\mathrm{H}}$, (b) $\mathrm{T}_{10: 12}^{\mathrm{H}}$, (c) $\mathrm{T}_{15: 18}^{\mathrm{H}}$, (d) $\mathrm{T}_{21: 25}^{\mathrm{H}}$, (e) $\mathrm{C}_{3: 3}^{\mathrm{H}}$, (f) $\mathrm{R}_{24: 24}^{\mathrm{H}}$, (g) $\mathrm{P}^{\prime} 18: 19$, (h) $\mathrm{S}_{19: 21}^{\mathrm{H}}$, and (i) $\mathrm{P}_{18: 21}^{\mathrm{H}}$; the peaks around Fermi level are marked with arrows. It can be seen that only the $p_{z}$ state contributes to the moment from $\left(c^{\prime}\right)$ projected density of states of one edge atom in the $\mathrm{T}_{15: 18}^{\mathrm{H}}$ GNM. 
upper left and upper rightsix-rings in Fig. 2(h). Even though the overall trend of the calculated values qualitatively follows the Lieb's theory, differences are observed, and first-principles calculations do not always correspond to $\Delta_{A B}$, even for the case with $A+B$ is even of $\mathrm{S}_{19: 21}^{\mathrm{H}}$ as we have already mentioned. In fact, we can ascribe the structures with an odd number of $A+B$ atoms, i.e., $\mathrm{T}_{6: 7}^{\mathrm{H}}, \mathrm{P}_{18: 19}^{\prime} \mathrm{H}, \mathrm{T}_{15: 18}^{\mathrm{H}}$, and $\mathrm{P}_{18: 21}^{\mathrm{H}}$ as well as sector shape $\mathrm{S}_{19: 21}^{\mathrm{H}}$ GNMs to intermediate regime between nonmagnetic and highly magnetic regimes. This regime provides a root toward the design of magnetic GNM supermeshes. It is worth noting that the possible mechanism for the deviation from the Lieb's theorem of the moment value for the sector shape GNM $S_{19: 21}^{\mathrm{H}}$ compared to $\mathrm{T}_{10: 12}^{\mathrm{H}}$ where $A+B$ is even for both, could be attributed to the larger amount of armchair edges (not favorable for moment formation) in the $\mathrm{S}_{19: 21}^{\mathrm{H}}$ structure.

To further elucidate the origin of magnetism in GNM structures, we compute the total and partial density of states (DOS) for considered GNMs. Figures 4(a)-4(d) give the total DOS for the triangular-shape GNMs shown in Figs. 2(a)-2(d), respectively. The exchange splitting between majority and minority spins mainly originates from $p_{z}$ orbitals, as clearly seen from Fig. $4\left(c^{\prime}\right)$ where the projected density of states (pDOS) on edge atoms for $\mathrm{T}_{15: 18}^{\mathrm{H}}$ is plotted. More interestingly, exchange splitting and energy differences between FM and PM states also increase with $\Delta_{A B}$ following the same trend as the net magnetic moment [see Figs. 3(a) and 3(b)],

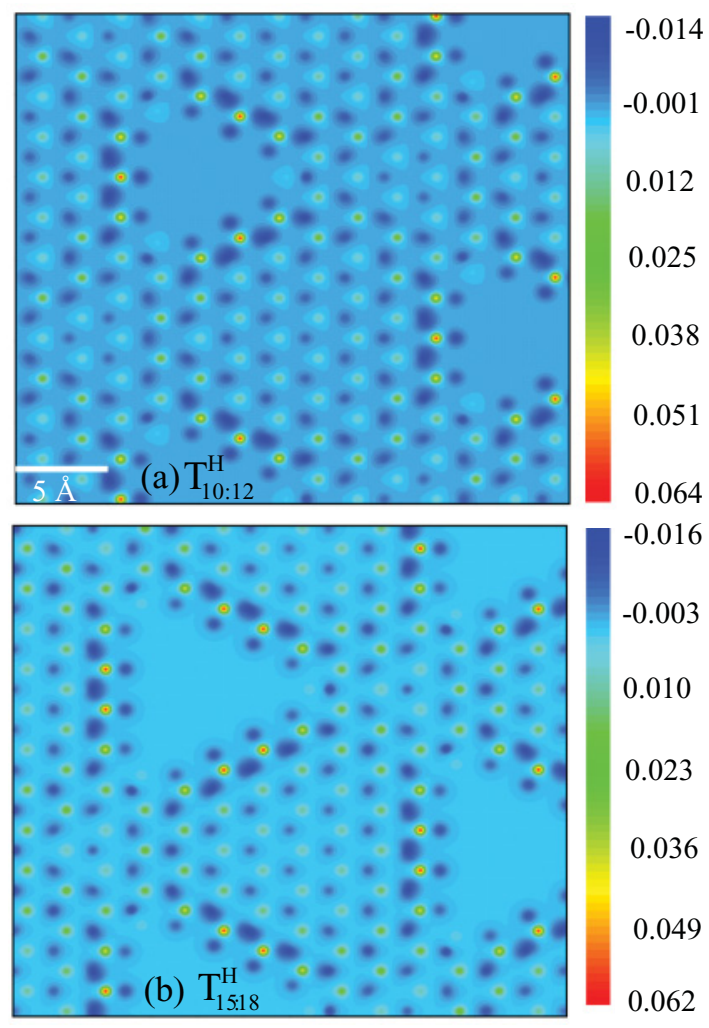

FIG. 5. (Color online) Spin density $\left(\mu_{B} / \AA^{2}\right)$ distribution for the two types of graphene nanomesh with localized (a) and delocalized (b) unpaired electrons. Localization and delocalization for these structures can be clearly seen in Fig. 4(b) and 4(c), respectively. reaching values of 0.5 and $0.12 \mathrm{eV}$, respectively. These large exchange-splitting values suggest that the magnetism could be preserved at room temperature, which looks very promising for room-temperature graphene spintronics developments. The density of states of structures like $\mathrm{T}_{10: 12}^{\mathrm{H}}$ provide evidence of the presence of localized electrons on the zigzag edges similar to the perfect GNR. ${ }^{18}$ The smearing of the $p_{z}$ peak and increase of the number of states at the Fermi level (metallization) suggest the electron delocalization on edges and switch from AFM to FM configuration similar to the case of partially oxidized graphite edges. ${ }^{49}$ We have plotted the spin-density figures for the localized and delocalized cases to illustrate dramatic changes in the distribution of electrons with vanishing of the pseudogap in density of states (see Fig. 5). The cause of such delocalization of the unpaired electrons on the edges of GNM holes can be attributed to a combination of sublattice degeneracy breaking and deviation from the perfect shapes of the graphene nanoribbon.

\section{CONCLUSIONS}

In conclusion, electronic and magnetic properties have been explored in GNM with different geometries using firstprinciples calculations. For balanced nonpassivated GNMs, the ground state was found to be either paramagnetic or antiferromagnetic. In situations of sublattice degeneracy breaking, ferrimagnetic ground states were obtained. The hydrogenpassivated GNMs were found to be strongly sensitive to the GNM size and shape, with magnetic moments deviation from the Lieb's theorem trend caused by the delocalization of the unpaired electrons on the zigzag edges. The calculations of the formation energy provide evidence of the structural stability and high probability of the formation of ferrimagnetic structures. Furthermore, three magnetic regimes are revealed: (i) highly magnetic GNM obeying Lieb's theorem; (ii) quenched magnetic state due to complete chemical bond reconstruction; and (iii) intermediate regime providing a possible root toward design of magnetic GNM supermeshes. These results demonstrate that a turn from zero-dimensional graphene nanoflakes throughout one-dimensional GNR with zigzag edges to GNM breaks localization of unpaired electrons at zigzag edges and provides a deviation from the Lieb's theorem trend. Such delocalization of the electrons allows switching of the ground state of systems from an antiferromagnetic narrow gap insulator discussed for GNRs to a ferromagnetic or nonmagnetic metal. These results combined with obtained large values of the exchange splitting (increasing with $\Delta_{A B}$ ) pinpoint promising perspectives for developing room-temperature graphene spintronics.

\section{ACKNOWLEDGMENTS}

We thank Xavier Blase, Albert Fert, Irina Grigorieva, Yakov Kopelevich, and Oleg Yazyev for fruitful discussions. This work was supported by Chair of Excellence Program of the Nanosciences Foundation in Grenoble, France, by French National Research Agency (ANR) Projects NANOSIMGRAPHENE and NMGEM, and by European Union funded STREP Project CONCEPT-GRAPHENE. 
*mair.chshiev@cea.fr

${ }^{1}$ K. S. Novoselov, A. K. Geim, S. V. Morozov, D. Jiang, Y. Zhang, S. V. Dubonos, I. V. Grigorieva, and A. A. Firsov, Science 306, 666 (2004).

${ }^{2}$ K. S. Novoselov, A. K. Geim, S. V. Morozov, D. Jiang, M. I. Katsnelson, I. V. Grigorieva, S. V. Dubonos, and A. A. Firsov, Nature (London) 438, 197 (2005).

${ }^{3}$ Y. Zhang, Y.-W. Tan, H. L. Stormer, and P. Kim, Nature (London) 438, 201 (2005).

${ }^{4}$ C. Berger, Z. Song, X. Li, X. Wu, N. Brown, C. Naud, D. Mayou, T. Li, J. Hass, A. N. Marchenkov, E. H. Conrad, P. N. First, and W. A. de Heer, Science 312, 1191 (2006).

${ }^{5}$ A. K. Geim and K. S. Novoselov, Nature Mater. 6, 183 (2007).

${ }^{6}$ M. I. Katsnelson, Mater. Today 10, 20 (2007).

${ }^{7}$ D. Huertas-Hernando, F. Guinea, and A. Brataas, Phys. Rev. B 74, 155426 (2006).

${ }^{8}$ Y. Yao, F. Ye, X.-L. Qi, S.-C. Zhang, and Z. Fang, Phys. Rev. B 75, 041401 (2007).

${ }^{9}$ A. Fert, A. Barthelemy, J. B. Youssef, J.-P. Contour, V. Cros, J. M. De Teresa, A. Hamzic, J. M. George, G. Faini, J. Grollier, H. Jaffres, H. Le Gall, F. Montaigne, F. Pailloux, and F. Petroff, Mater. Sci. Eng., B 84, 1 (2001).

${ }^{10}$ S. A. Wolf, D. D. Awschalom, R. A. Buhrman, J. M. Daughton, S. von Molnar, M. L. Roukes, A. Y. Chtchelkanova, and D. M. Treger, Science 294, 1488 (2001).

${ }^{11}$ N. Tombros, C. Jozsa, M. Popinciuc, H. T. Jonkman, and B. J. van Wees, Nature (London) 448, 571 (2007).

${ }^{12}$ W. Han, W. H. Wang, K. Pi, K. M. McCreary, W. Bao, Y. Li, F. Miao, C. N. Lau, and R. K. Kawakami, Phys. Rev. Lett. 102, 137205 (2009).

${ }^{13}$ W. Han, K. Pi, K. M. McCreary, Y. Li, J. J. I. Wong, A. G. Swartz, and R. K. Kawakami, Phys. Rev. Lett. 105, 167202 (2010).

${ }^{14}$ T.-Y. Yang, J. Balakrishnan, F. Volmer, A. Avsar, M. Jaiswal, J. Samm, S. R. Ali, A. Pachoud, M. Zeng, M. Popinciuc, G. Güntherodt, B. Beschoten, and B. Özyilmaz, Phys. Rev. Lett. 107, 047206 (2011).

${ }^{15}$ B. Dlubak, P. Seneor, A. Anane, C. Barraud, C. Deranlot, D. Deneuve, B. Servet, R. Mattana, F. Petroff, and A. Fert, Appl. Phys. Lett. 97, 092502 (2010).

${ }^{16}$ Y.-W. Son, M. L. Cohen, and S. G. Louie, Phys. Rev. Lett. 97, 216803 (2006).

${ }^{17}$ L. Yang, C. H. Park, Y.-W. Son, M. L. Cohen, and S. G. Louie, Phys. Rev. Lett. 99, 186801 (2007).

${ }^{18}$ Y.-M. Son, M. L. Cohen, and S. G. Louie, Nature (London) 444, 347 (2006).

${ }^{19}$ L. Brey, H. A. Fertig, and S. Das Sarma, Phys. Rev. Lett. 99, 116802 (2007); M. Wimmer, I. Adagideli, S. Berber, D. Tomanek, and K. Richter, ibid. 100, 177207 (2008); F. Muñoz-Rojas, J. FernàndezRossier, and J. J. Palacios, ibid. 102, 136810 (2009).

${ }^{20}$ O. V. Yazyev and L. Helm, Phys. Rev. B 75, 125408 (2007); O. V. Yazyev, ibid. 101, 037203 (2008); Rep. Prog. Phys. 73, 056501 (2010).

${ }^{21}$ J. Fernández-Rossier and J. J. Palacios, Phys. Rev. Lett. 99, 177204 (2007); J. J. Palacios, J. Fernández-Rossier, and L. Brey, Phys. Rev. B 77, 195428 (2008).

${ }^{22}$ T. G. Pedersen, C. Flindt, J. Pedersen, N. A. Mortensen, A. P. Jauho, and K. Pedersen, Phys. Rev. Lett. 100, 136804 (2008).
${ }^{23}$ J. A. Furst, T. G. Pedersen, M. Brandbyge, and A.-P. Jauho, Phys. Rev. B 80, 115117 (2009).

${ }^{24}$ X. H. Zheng, G. R. Zhang, Z. Zeng, V. M. García-Suárez, and C. J. Lambert, Phys. Rev. B 80, 075413 (2009).

${ }^{25}$ D. Yu, E. M. Lupton, M. Liu, W. Liu, and F. Liu, Nano Res. 1, 56 (2008).

${ }^{26}$ H. Y. He, Y. Zhang, and B. C. Pan, J. Appl. Phys. 107, 114322 (2010).

${ }^{27}$ L. Chen, D. Yu, and F. Liu, Appl. Phys. Lett. 93, 223106 (2008).

${ }^{28}$ P. O. Lehtinen, A. S. Foster, Y. Ma, A. V. Krasheninnikov, and R. M. Nieminen, Phys. Rev. Lett. 93, 187202 (2004).

${ }^{29}$ Nicolas Leconte, David Soriano, Stephan Roche, Pablo Ordejon, Jean-Christophe Charlier, and J. J. Palacios, ACS Nano 5, 3987 (2011).

${ }^{30}$ David Soriano, Nicolas Leconte, Pablo Ordejón, Jean-Christophe Charlier, Juan-Jose Palacios, and Stephan Roche, Phys. Rev. Lett. 107, 016602 (2011).

${ }^{31}$ P. Esquinazi, D. Spemann, R. Höhne, A. Setzer, K.-H. Han, and T. Butz, Phys. Rev. Lett. 91, 227201 (2003); J. Cervenka, M. I. Katsnelson, and C. F. J. Flipse, Nat. Phys. 5, 840 (2009); D. Martinez-Martin, M. Jaafar, R. Perez, J. Gomez-Herrero, and A. Asenjoar, Phys. Rev. Lett. 105, 257203 (2010); M. M. Ugeda, I. Brihuega, F. Guinea, and J. M. Gómez-Rodríguez, Phys. Rev. Lett. 104, 096804 (2010); H. Ohldag, P. Esquinazi, E. Arenholz, D. Spemann, M. Rothermel, A. Setzer, and T. Butz, New J. Phys. 12, 123012 (2010); M. Sepioni, R. R. Nair, S. Rablen, J. Narayanan, F. Tuna, R. Winpenny, A. K. Geim, and I. V. Grigorieva, Phys. Rev. Lett. 105, 207205 (2010).

${ }^{32}$ D. W. Boukhvalov, Phys. Chem. Chem. Phys. 12, 15367 (2010); D. W. Boukhvalov and M. I. Katsnelson, ACS Nano 5, 2440 (2011).

${ }^{33}$ O. V. Yazyev and M. I. Katsnelson, Phys. Rev. Lett. 100, 047209 (2008).

${ }^{34}$ J. Č ervenka, M. I. Katsnelson, and C. F. J. Flipse, Nat. Phys. 5, 840 (2009); T. Enoki and K. Takai, Solid. State. Commun. 149, 1144 (2009).

${ }^{35}$ K. Tada, J. Haruyama, H. X. Yang, M. Chshiev, T. Matsui, and H. Fukuyama, Phys. Rev. Lett. 107, 217203 (2011).

${ }^{36}$ F. Banhart, J. Kotakoski, and A. V. Krasheninnikov, ACS Nano 5, 26 (2011)

${ }^{37}$ O. V. Yazyev, W. L. Wang, S. Meng, and E. Kaxiras, Nano Lett. 8, 766 (2008); W. L. Wang, O. V. Yazyev, S. Meng, and E. Kaxiras, Phys. Rev. Lett. 102, 157201 (2009).

${ }^{38}$ A. Cresti and S. Roche, New J. Phys. 11, 095004 (2009); A. C. Ferrari, Solid State Commun. 143, 47 (2007).

${ }^{39}$ S. Wang and M. Grifoni, Phys. Rev. Lett. 95, 266802 (2005); E. H. Hwang, Ben Yu-Kuang Hu, S. Das Sarma, ibid. 99, 226801 (2007).

${ }^{40}$ J. Bai, X. Zhong, S. Jiang, Y. Huang, and X. Duan, Nat. Nanotechnol. 5, 190 (2010).

${ }^{41}$ E. H. Lieb, Phys. Rev. Lett. 62, 1201 (1989).

${ }^{42}$ Z. Shi, R. Yang, L. Zhang, Y. Wang, D. Liu, D. Shi, E. Wang, and G. Zhang, Adv. Mater. 23, 3061 (2011).

${ }^{43}$ In some literature, ferrimagnetic states described in this work have been called ferromagnetic based on definitions considering only one sublattice. 
${ }^{44}$ G. Kresse and J. Hafner, Phys. Rev. B 47, 558 (1993); 49, 14251 (1994); G. Kresse and J. Furthmuller, Comput. Mater. Sci. 6, 15 (1996); Phys. Rev. B 54, 11169 (1996).

${ }^{45}$ P. E. Blochl, Phys. Rev. B 50, 17953 (1994).

${ }^{46}$ J. P. Perdew, K. Burke, and M. Ernzerhof, Phys. Rev. Lett. 77, 3865 (1996).
${ }^{47}$ M. Methfessel and A. T. Paxton, Phys. Rev. B 40, 3616 (1989).

${ }^{48}$ D. W. Boukhvalov and M. I. Katsnelson, Nano Lett. 8, 4373 (2008).

${ }^{49}$ D. W. Boukhvalov, S. Moehlecke, R. R. da Silva, and Y. Kopelevich, Phys. Rev. B 83, 233408 (2011). 